\title{
Schumann resonance parameters at Kuju station during solar flares
}

\author{
Akihiro Ikeda ${ }^{1,1}$, Teiji Uozumi ${ }^{2}$, Akimasa Yoshikawa ${ }^{2}$, Akiko Fujimoto $^{3}$, and Shuji Abe $^{2}$ \\ ${ }^{1}$ National Institute of Technology, Kagoshima College, Japan \\ ${ }^{2}$ International Center for Space Weather Science and Education, Kyushu University, Japan \\ ${ }^{3}$ Kyushu Institute of Technology, Japan
}

\begin{abstract}
We examined the Schumann resonance (SR) at low-latitude station KUJ by comparing with solar X-ray flux and solar proton flux at a geostationary orbit. For intense solar activity in October-November 2003, the reaction of the SR frequency to X-ray enhancement and SPEs was different. The SR frequency in $\mathrm{H}$ component increased at the time of the $\mathrm{X}$ ray enhancement. The response of SR seems to be caused by the increase of the electron density in the ionospheric $\mathrm{D}$ region which ionized by the enhanced solar X-ray flux. In the case of the SPEs, the SR frequency in D component decreased with enhancement of solar proton flux. We suggest that the SPEs caused the decrease of altitude on the ionopheric D region at high-latitude region, and the SR frequency decreased.
\end{abstract}

\section{Introduction}

Solar flare is the extraordinary activity of the sun. At the time of solar flares, the solar X-ray flux increases and is often followed by solar proton events (SPEs). Such activities have affects on the Earth's ionosphere. Enhanced solar X-ray flux increases the electron density and changes the altitude profile of its from the ionospheric $\mathrm{D}$ region up to the $\mathrm{F}$ region [1]. If SPE subsequently occurs, high-energy solar protons penetrate deep into the ionosphere and the atmosphere. Solar protons with energies of several $\mathrm{MeV}$ cause drastic changes in the ionospheric D-region. Especially, SPE affects the whole of the high-latitude regions more than 60 degree geomagnetic latitude [2].

The Schumann resonance (SR) is the global resonance of electromagnetic waves generated by global lightning activity. SR parameters, which are amplitude and frequency, reflect the properties of both global lightning activity and the state of the Earth-ionosphere cavity [3]. Thus, it was revealed that the SR is also affected by the solar activities [4]. We newly aim to utilize SR for monitoring of the ionospheric D region by using our ground magnetic field data. For the first step, we investigated relationship between SR and intense solar activities in October-November 2003.

${ }^{1}$ Corresponding author: a-ikeda@kagoshima-ct.ac.jp 


\section{Data Set}

We examined the fundamental mode of SR observed by an induction magnetometer at Kuju, Japan (M.Lat. = 23.4 degree, M. Lon. = 201.0 degree). The observation is a part of activities by International Center for Space Weather Science and Education, Kyushu University and the observation of the induction magnetometer started in 2003. The components of ground magnetic field used in this study are horizontal northward component $(\mathrm{H})$ and horizontal eastward component $(\mathrm{D})$. Sampling rate of the observation data is $50 \mathrm{~Hz}$.

We identified frequency of the SR by calculating PSD (power spectral density) every 10 seconds segment by using FFT (First Fourier Transformation). Generally, frequency of the fundamental mode of SR is about $7.8 \mathrm{~Hz}$ [5], we therefore found a peak frequency ranging $6.0 \mathrm{~Hz}$ to $9.0 \mathrm{~Hz}$. This peak frequency is denoted by SR frequency.

To compare with SR frequency, solar X-ray (0.05-0.3 nm) and proton flux (40-80 MeV) were also analyzed. These data were obtained by the GOES-10 satellite on a geostationary orbit.

\section{Results and Discussion}

The upper panel of Figure 1 shows the solar X-ray flux from 14 October to 9 November in 2003. The subsequent panels in Figure 1 show the corresponding time history of the SR frequency in $\mathrm{H}$ and $\mathrm{D}$ components.

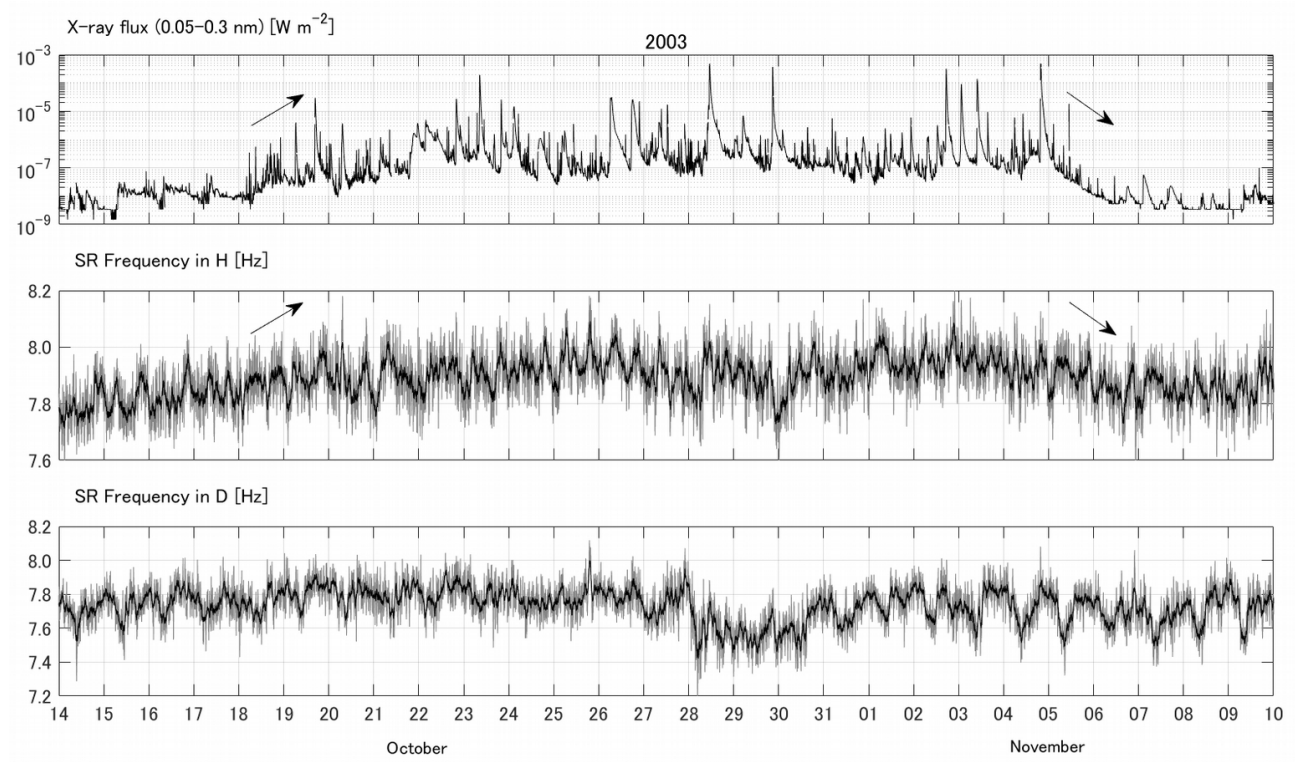

Fig. 1. Variation of the X-ray flux $(0.05-0.3 \mathrm{~nm})$, SR frequency in $H$, and SR frequency in $D$. The grey and black lines for SR frequencies in $\mathrm{H}$ and $\mathrm{D}$ components are 100 and 500 point-centered running averages of the data.

The enhancement of solar X-ray flux occurred on 18 October, 2003 and lasted for about 19 days before it recovered to a previous level. The SR frequency in $\mathrm{H}$ component followed the variation of the X-ray flux (see black arrows in Figure 1). The effect on SR frequency is 
not related to the local time at the observatory, since a high degree of the SR frequency in $\mathrm{H}$ component continued during the enhancement of solar X-ray flux. The effect on SR seems to be the global character.

According to Roludugin et al. [6], two parameters of the ionospheric D region link with the SR frequency. One is an electron density, and the other is altitude of the lower boundary of the $\mathrm{D}$ region.

$$
\frac{\operatorname{Re}\left(\delta f_{1}\right)}{\operatorname{Re} f_{1}} \approx 0.36\left(\frac{\delta N}{2 N}+\frac{\delta h}{h}\right)
$$

The left side is the variation of the fundamental mode of the SR frequency. The first and second on the right side are the variation of the electron density and the altitude of the D region's lower edge, respectively. De et al. [7] suggested that the solar X-ray flux increases electron density without a significant change in the ionospheric altitude. Our results therefore imply that the increase of the SR frequency in $\mathrm{H}$ was caused by the enhancement of electron density in the ionospheric $\mathrm{D}$ region. In addition, it seems that the enhancement of electron density lasted for about 19 days.

Roludugin et al., [6] showed that the increase of the SR frequency in D component was prominent at the time of X-ray flare. For our event, the SR frequency in H component was more sensitive to the enhancement of X-ray than D component. This discrepancy is most likely due to the difference of the geographical location of observatories. We analyzed the data obtained at a low-latitude station, while Roludugin et al. analyzed the ground magnetic field data at high-latitude station Lovezero (68.0 degree north latitude ).

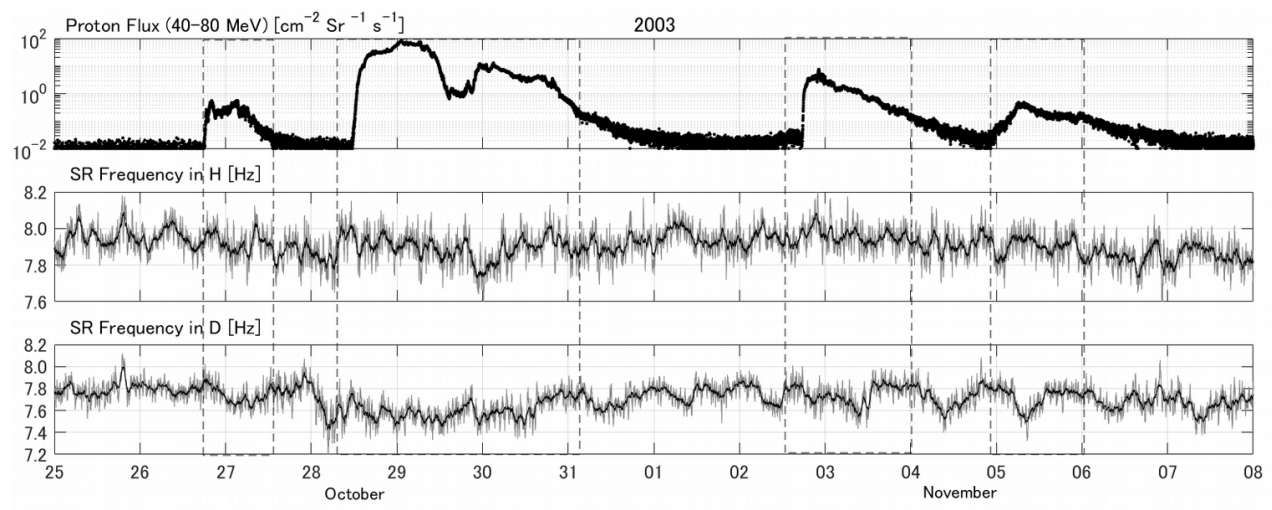

Fig. 2. Variation of the solar proton flux $(40-80 \mathrm{MeV})$, SR frequency in $\mathrm{H}$, and SR frequency in D. The time interval of Figure 2 is different from that of Figure 1.

Figure 2 shows the solar proton flux in $40-80 \mathrm{MeV}$ from 25 October to 7 November in 2003. We note that the time interval of Figure 2 is different from that of Figure 1. During the enhancement of the X-ray on 18 October to 6 November in 2003 (see Figure 1), the prominent SPEs occurred several times as the enhancement of the proton flux. They started on 26 October, 28 October, 2 November, and 5 November, 2003 (see Figure 2). The dotted rectangles in Figure 2 show the SPEs. All of these SPEs were accompanied by the decreases of the SR frequency in D component. This tendency is not clear in H component.

Similarly, Roldugin et al. [6] found a decrease of SR frequency at high-latitude station during a SPE, and suggested that the variation of the SR frequency was caused by a decrease of the ionospheric altitude at high latitudes. De et al. [7] pointed out that SPE can 
decrease/increase SR frequency since the solar protons cause a decrease of altitude of the ionospheric $\mathrm{D}$ region and also affects ionization. Thus two terms compete with each other and decrease/increase SR frequency. Threfore the decrease of SR frequency in D component for our SPEs were probably caused by the decrease of altitude on the ionospheric D region in high-latitude region. We also suggest that the SR frequency in D component is more relevant to high-latitude ionosphere than that in $\mathrm{H}$ component.

In conclusion, we examined the SR at low-latitude station KUJ for intense solar activity in Octover-November, 2003 and found that the reaction of the SR frequency to X-ray enhancement and SPE is different. The SR frequency in H component increased at the time of the X-ray enhancement. This seems to be caused by the increase of the electron density in the ionospheric D region which ionized by the intense solar X-ray flux. In the case of the SPEs, the SR frequency in D component decreased with enhancement of solar proton flux. The decrease of the SR frequency are probably caused by the decrease of altitude on the ionopheric $\mathrm{D}$ region in high-latitude region. In the future, we need to study these events in a quantitative way.

The study was funded by The Mazda Foundation (2017). The induction magnetic field data is taken by International Center for Space Weather Science and Education, Kyushu University. The solar X-ray and proton flux data are taken by GOES-10 satellite.

\section{References}

1. Thome, G. D., L. S. Wagner, J. Geophys. Res., 76, 6,883 (1971)

2. Reagan, J. B., T. M. Watt, J. Geophys. Res., 81, 4,579 (1971)

3. Sentmanm D., D. J. Fraser, J. Geophys. Res., 95, 15,973 (1991)

4. Sao, K., M. Yamashita, S. Tanahashi, H. Jindo, K. Ohta, J. Atmos. Terr. Phys., 35, 2,047 (1973)

5. Balser, M., C. A. Wagner, J. Geophys. Res., 67, 4,081 (1962)

6. Roldugin, V. C., Ye. P. Maltsev, A. N. Vasiljev, E. V. Vashenyuk, Ann. Geophysicae, 17, 1,293 (1999)

7. De, S. S., B. K. De, B. Bandyopadhyay, Suman Paul, D. K. Haldar, S. Barui, Journal of Atmospheric and Solar-Terrestrial Physics, 72, 829 (2010) 